\title{
Resultados del servicio de farmacovigilancia en una farmacia comunitaria
}

\author{
Damià Barris Blundell(D, Belén Sabio Sánchez, Rocío Sánchez Gómez, Gemma Benítez Pacheco, \\ María Isabel Compaña Ariza ${ }^{-}$, Elena Navarro Visa $\odot$ \\ Farmacéuticos comunitarios en Farmacia Zarzuelo, Benalmádena (Málaga).
}

\section{PALABRAS CLAVE}

Farmacovigilancia, farmacia comunitaria, reacciones adversas a medicamentos, monitorización de fármacos, tarjeta amarilla

\section{ABREVIATURAS}

RAM: Reacciones adversas a medicamentos

PRM: Problemas relacionados con medicamentos

\section{KEYWORDS}

Pharmacovigilance, community pharmacy, adverse drug reactions, drug follow-up, yellow card

\section{RESUMEN}

Introducción: el sistema español de farmacovigilancia se basa en la notificación espontánea de sospechas de Reacciones Adversas a Medicamentos (RAM) por parte de los pacientes, la industria farmacéutica y los profesionales sanitarios mediante la tarjeta amarilla. La farmacovigilancia es una de las actividades que puede desarrollar la farmacia comunitaria y que, además, está contemplada en la legislación como una labor obligatoria para todos los profesionales sanitarios. El objetivo de este artículo es describir y analizar las sospechas de RAM detectadas por el equipo farmacéutico de una farmacia comunitaria y comunicadas al Centro Andaluz de Farmacovigilancia desde el año 2002 al año 2018.

Métodos: estudio observacional longitudinal realizado en una farmacia comunitaria de Benalmádena (Málaga) con las sospechas de RAM detectadas y notificadas mediante tarjeta amarilla al Centro Andaluz de Farmacovigilancia desde el año 2002 hasta el año 2018.

Resultados: el número total de sospechas de RAM comunicadas al Centro Andaluz de Farmacovigilancia desde el año 2002 ha sido de 329. La edad media de los pacientes que presentaron una RAM ha sido de 59,3 años (DE: 16,8). Los grupos farmacológico implicados en las RAM con una mayor frecuencia han sido los agentes modificadores de los lípidos (9,4 \%), agentes que actúan sobre el sistema renina-angiotensina (9,4\%), antibacterianos (6,9\%), antiinflamatorios $(6,9 \%)$ y psicoanalépticos $(6,4 \%)$. Las RAM detectadas han afectado principalmente al sistema nervioso (31,7 \%), al tracto gastrointestinal $(22,4 \%)$ y a la piel $(14,3 \%)$.

Conclusiones: más de la mitad de las sospechas comunicadas se han registrado en pacientes mayores de 65 años. El equipo farmacéutico debe seguir atento a la detección de sospechas de RAM en este grupo de pacientes, especialmente en los medicamentos de nueva comercialización.

El análisis de las sospechas de RAM detectadas permite disponer de un conocimiento más completo de la seguridad del medicamento en nuestra actividad.

\section{Results of pharmacovigilance in a community pharmacy}

\section{ABSTRACT}

Introduction: The spanish pharmacovigilance system is based on spontaneous notification of suspected Adverse Drug Reactions (ADRs) by patients, the pharmaceutical industry and healthcare professionals using the yellow card. Pharmacovigilance is one of the activities that the community pharmacy can develop and that, in addition, is contemplated in the legislation as a mandatory task for all healthcare professionals.

The objective of this article is to describe and analyze the suspected ADRs detected by the pharmacists of a community pharmacy and reported to the Andalusian Center for Pharmacovigilance from 2002 to 2018.

Methods: Observational longitudinal study carried out in a community pharmacy in Benalmádena (Málaga) with suspected ADR detected and notified by yellow card to the Andalusian Center for Pharmacovigilance from 2002 to 2018.

Results: The total number of suspicions of ADR reported to the Andalusian Center for Pharmacovigilance since 2002 has been 329. The average age of the patients who presented ADR was 59.3 years (SD: 16.8). The pharmacological groups most frequently involved in ADRs have been lipid modifying agents (9.4\%), agents that act on the renin-angiotensin system (9.4\%), antibacterials (6.9\%), antiinflammatories (6.9\%) and psycholeptics (6.4\%). The detected ADRs have mainly affected the nervous system (31.7\%), the gastrointestinal tract (22.4\%) and the skin (14.3\%).

Conclusions: More than half of the reported suspicions have been registered in patients over 65 years of age. The pharmaceutical team must remain vigilant in detecting suspected ADRs in this group of patients, especially in newly marketed drugs.

The analysis of the suspicions of ADR detected allows us to have a more complete knowledge of the safety of the medication in our activity.
Recibido: 20/5/2020 Aceptado: $20 / 7 / 2020$

Disponible online: 13/10/2020
Financiación: ninguna.

Conflicto de intereses: ninguno.

Cite este artículo como: Barris D, Sabio B, Sánchez R, Benitez G, Compaña MI, Navarro E. Resultados del servicio de farmacovigilancia en una farmacia comunitaria. Farmacéuticos Comunitarios. 2020 0ct 13; 12 (4): 30-36. doi:10.33620/ FC.2173-9218.(2020/Vol12).004.04

Correspondencia: Damià Barris Blundell (damiabarris@gmail.com).

ISSN 1885-8619 OSEFAC (Sociedad Española de Farmacia Familiar y Comunitaria). Todos los derechos reservados. 


\section{Introducción}

El estudio de la seguridad de un medicamento no finaliza con su comercialización, sino que es necesario analizar las reacciones adversas, especialmente en los primeros años en el mercado, para definir el perfil de seguridad del nuevo medicamento en la práctica clínica (1). Los estudios efectuados durante la investigación y el desarrollo de un nuevo medicamento facilitan un buen conocimiento de su calidad y eficacia; sin embargo, existen limitaciones para conocer su seguridad. La polimedicación, las pluripatologías y el comportamiento de los pacientes pueden influir en la reacción del organismo ante la administración de un nuevo medicamento. Muchas de las reacciones adversas a medicamentos (RAM), especialmente las de baja incidencia se manifiestan principalmente cuando su difusión alcanza un número elevado de pacientes. Su utilización en la población general y las condiciones de la práctica habitual permiten conocer con mayor precisión su perfil de seguridad $(2,3)$.

Las RAM son una causa frecuente, aunque a menudo prevenible, de morbimortalidad y de aumento del consumo de recursos sanitarios, con un gran impacto económico. En España, según el "Estudio sobre la seguridad de los pacientes en atención primaria" (APEAS) el $37 \%$ de las causas graves de consulta en centros de atención primaria están relacionadas con la medicación (4). Ocurre en el $10 \%$ de los pacientes ambulatorios, causan el 5-10 \% de los ingresos hospitalarios y las presentan el 10-20\% de los pacientes hospitalizados, lo que aumenta su estancia media (5). La incidencia de pacientes hospitalizados fallecidos por RAM en nuestro ámbito es del $7 \%$ (6). Por todo esto, las RAM son consideradas como un importante problema para la salud pública, que afecta al conjunto de la población y sobre el que hay que intervenir.

En nuestro país existe un sistema de farmacovigilancia para facilitar la recogida de información sobre los efectos adversos que pueden ocasionar los medicamentos. La farmacovigilancia, como actividad de salud pública, se responsabiliza de recoger, elaborar y, en su caso, procesar la información sobre sospechas de RAM, que identifican los profesionales sanitarios o los ciudadanos, con la finalidad de identificar riesgos previamente no conocidos o cambios de riesgos ya conocidos, así como para la realización de cuantos estudios se consideren necesarios para confirmar y/o cuantificar dichos riesgos (7).

El sistema español de farmacovigilancia se basa en la notificación espontánea de sospechas de RAM por parte de los pacientes, la industria farmacéutica y los profesionales sanitarios mediante la tarjeta amarilla. Entre las ventajas de este sistema de notificación cabe destacar varios aspectos: se trata de un método que precisa escasa cantidad de recursos para su funcionamiento, permite el estudio permanente de un amplio número de pacientes y posibilita detectar de forma precoz la aparición de un efecto indeseable inesperado $(3,8)$.

La farmacovigilancia es una de las actividades que puede desarrollar la farmacia comunitaria y que, además está contemplada en la legislación como una labor obligatoria para todos los profesionales sanitarios. El papel del farmacéutico en la farmacovigilancia está regulado por varias normas: la Ley 44/2003 de 21 de noviembre, de ordenación de las profesiones sanitarias (9), la Ley 29/2006, de 29 de julio, de garantías y uso racional de los medicamentos y productos sanitarios (10) y el Real Decreto 577/2013, de 27 de julio, por el que se regula la farmacovigilancia de medicamentos de uso humano (7).

El objetivo de este artículo es describir y analizar las sospechas de RAM detectadas por el equipo farmacéutico de una farmacia comunitaria y comunicadas al Centro Andaluz de Farmacovigilancia desde el año 2002 al año 2018.

\section{Métodos}

Estudio observacional longitudinal realizado en una farmacia comunitaria de Benalmádena (Málaga) con las RAM detectadas y notificadas mediante tarjeta amarilla al Centro Andaluz de Farmacovigilancia desde el año 2002 hasta el año 2018. El equipo está formado por cinco farmacéuticos comunitarios.

Durante la dispensación se ha seguido la sistemática descrita en el protocolo de la figura 1. Ante la sospecha de una RAM se procede a notificarla, previo consentimiento del paciente, especialmente las de medicamentos de reciente introducción, las graves y raras.

Se han recogido los siguientes datos:

- Número de tarjetas amarillas notificadas.

- Sistemas implicados en las RAM.

- Reacciones adversas.

- Sexo y edad del paciente.

- Fármacos sospechosos de haber causado las RAM.

Disponemos de dos posibilidades de notificación:

- Tarjeta amarilla en papel.

- Tarjeta amarilla on line.

En ambos formatos se recogen los datos mínimos necesarios para evaluar una posible relación de causalidad entre un medicamento y una RAM por parte del Centro Andaluz de Farmacovigilancia (tabla 1).

Los datos han sido procesados y analizados mediante el programa Excel ${ }^{\circledR}$. Los datos cualitativos se expresan como porcentajes y los cuantitativos como media y desviación estándar.

\section{Resultados}

El número total de RAM comunicadas al Centro Andaluz de Farmacovigilancia desde el año 2002 ha sido de 329. En la figura 2 se muestra el número de sospechas de RAM detectadas por año.

La edad media de los pacientes que presentaron una RAM ha sido de 59,3 años (DE: 16,8), con un rango de edad amplio, que se ha situado entre 1 año y 93 años. El número medio de medicamentos utilizados por paciente ha sido de 3,7 (DE: 2,2), con un rango que se ha situado entre 1 y 11 medicamentos. En relación al sexo de los pacientes, un 33,1\% $(n=109)$ han sido hombres y un 66,9\% $(n=220)$ mujeres.

Los fármacos implicados en las RAM con una mayor frecuencia han sido los agentes modificadores de los lípidos (C10), agentes que actúan sobre el sistema renina-angiotensina (C09), antibacterianos (J01), antiinflamatorios (M01) y psicoanalépticos (N06) (tabla 2).

Las RAM más frecuentes según los órganos y aparatos afectados han correspondido al sistema nervioso, sistema digestivo, piel y sistema músculo-esquelético (tabla 3). 


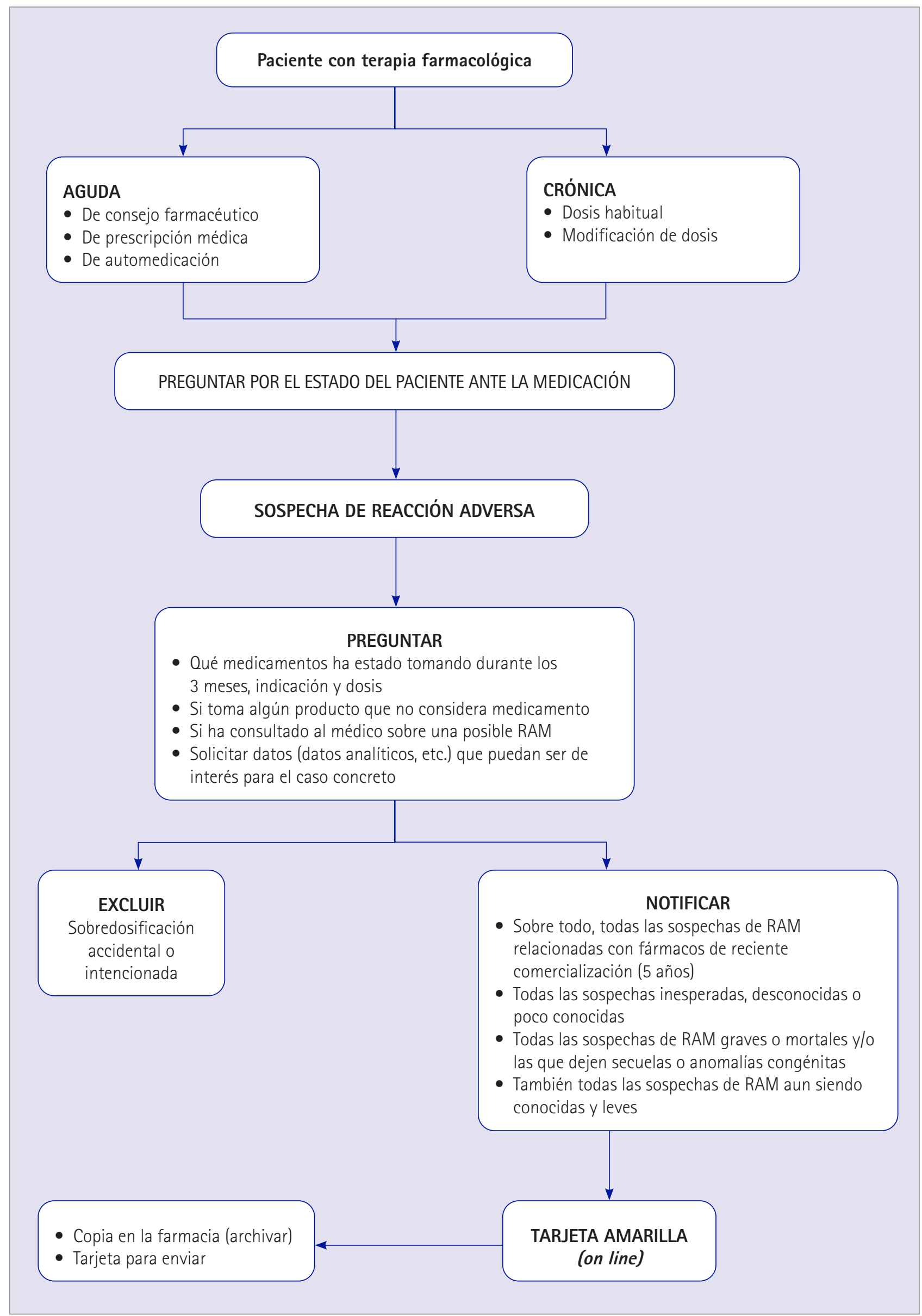

Figura 1 Protocolo de detección y comunicación de sospecha de RAM 
Tabla 1 Datos recogidos en la tarjeta amarilla

\begin{tabular}{|l|l|}
\hline Dato & Objeto \\
\hline Nombre del paciente & $\begin{array}{l}\text { Identifica RAM repetidas } \\
\text { o en un mismo individuo. } \\
\text { Farmacogenética. }\end{array}$ \\
\hline Edad del paciente & Identifica grupos de riesgo \\
\hline $\begin{array}{l}\text { Fármaco (nombre } \\
\text { comercial) }\end{array}$ & $\begin{array}{l}\text { Detección de RAM causadas } \\
\text { por el excipiente, forma } \\
\text { farmacéutica, etc. }\end{array}$ \\
\hline $\begin{array}{l}\text { Dosis diaria y vía de } \\
\text { administración }\end{array}$ & $\begin{array}{l}\text { Relación temporal de reacciones } \\
\text { adversas en politerapia. }\end{array}$ \\
\hline $\begin{array}{l}\text { Cronología de } \\
\text { exposición al } \\
\text { fármaco }\end{array}$ & $\begin{array}{l}\text { Imputa la RAM al fármaco. } \\
\text { Descartar patología causal } \\
\text { alternativa. }\end{array}$ \\
\hline $\begin{array}{l}\text { Motivo de la } \\
\text { prescripción }\end{array}$ & $\begin{array}{l}\text { Orienta la patología base. Puede } \\
\text { contribuir a la aparición de una } \\
\text { RAM. }\end{array}$ \\
\hline $\begin{array}{l}\text { Desenlace del } \\
\text { acontecimiento }\end{array}$ & $\begin{array}{l}\text { Permite establecer la gravedad } \\
\text { notifica }\end{array}$ \\
\hline $\begin{array}{l}\text { Observaciones } \\
\text { adicionales }\end{array}$ & $\begin{array}{l}\text { Información clínica o analitica } \\
\text { para precisar la imputabilidad de } \\
\text { la RAM. }\end{array}$ \\
\hline centro \\
\hline Profesional que
\end{tabular}

Tabla 2. Grupos farmacológicos implicados en las sospechas de RAM

\begin{tabular}{|c|c|}
\hline Grupos farmacológicos & n (\%) \\
\hline $\begin{array}{l}\text { Co9 Agentes que actúan sobre el sistema } \\
\text { renina-angiotensina }\end{array}$ & $31(9,4 \%)$ \\
\hline C10 Agentes modificadores de los lípidos. & $31(9,4 \%)$ \\
\hline J01 Antibacterianos para uso sistémico & $23(6,9 \%)$ \\
\hline $\begin{array}{l}\text { M01 Productos antiinflamatorios y } \\
\text { antirreumáticos }\end{array}$ & $23(6,9 \%)$ \\
\hline N06 Psicoanalépticos & $21(6,4 \%)$ \\
\hline G04 Productos de uso urológico & $20(6,1 \%)$ \\
\hline N02 Analgésicos & $16(4,9 \%)$ \\
\hline S01 Oftalmológicos & $15(4,6 \%)$ \\
\hline $\begin{array}{l}\text { M05 Fármacos para el tratamiento de } \\
\text { enfermedades óseas }\end{array}$ & $12(3,6 \%)$ \\
\hline N03 Antiepilépticos & $11(3,3 \%)$ \\
\hline N05 Psicolépticos & $10(3,0 \%)$ \\
\hline A10 Fármacos usados en diabetes & $10(3,0 \%)$ \\
\hline $\begin{array}{l}\text { R03 Agentes contra padecimientos } \\
\text { obstructivos de las vías respiratorias }\end{array}$ & $9(2,7 \%)$ \\
\hline $\begin{array}{l}\text { A02 Agentes para el tratamiento de } \\
\text { alteraciones causadas por ácidos }\end{array}$ & $8(2,4 \%)$ \\
\hline R06 Antihistamínicos para uso sistémico & $7(2,1 \%)$ \\
\hline $\begin{array}{l}\text { A03 Agentes contra padecimientos funcionales } \\
\text { del estómago e intestino }\end{array}$ & $5(1,5 \%)$ \\
\hline B01 Agentes antitrombóticos & $5(1,5 \%)$ \\
\hline C08 Bloqueantes de canales de calcio & $5(1,5 \%)$ \\
\hline R01 Preparados de uso nasal & $5(1,5 \%)$ \\
\hline R05 Preparados para la tos y el resfriado & $5(1,5 \%)$ \\
\hline
\end{tabular}

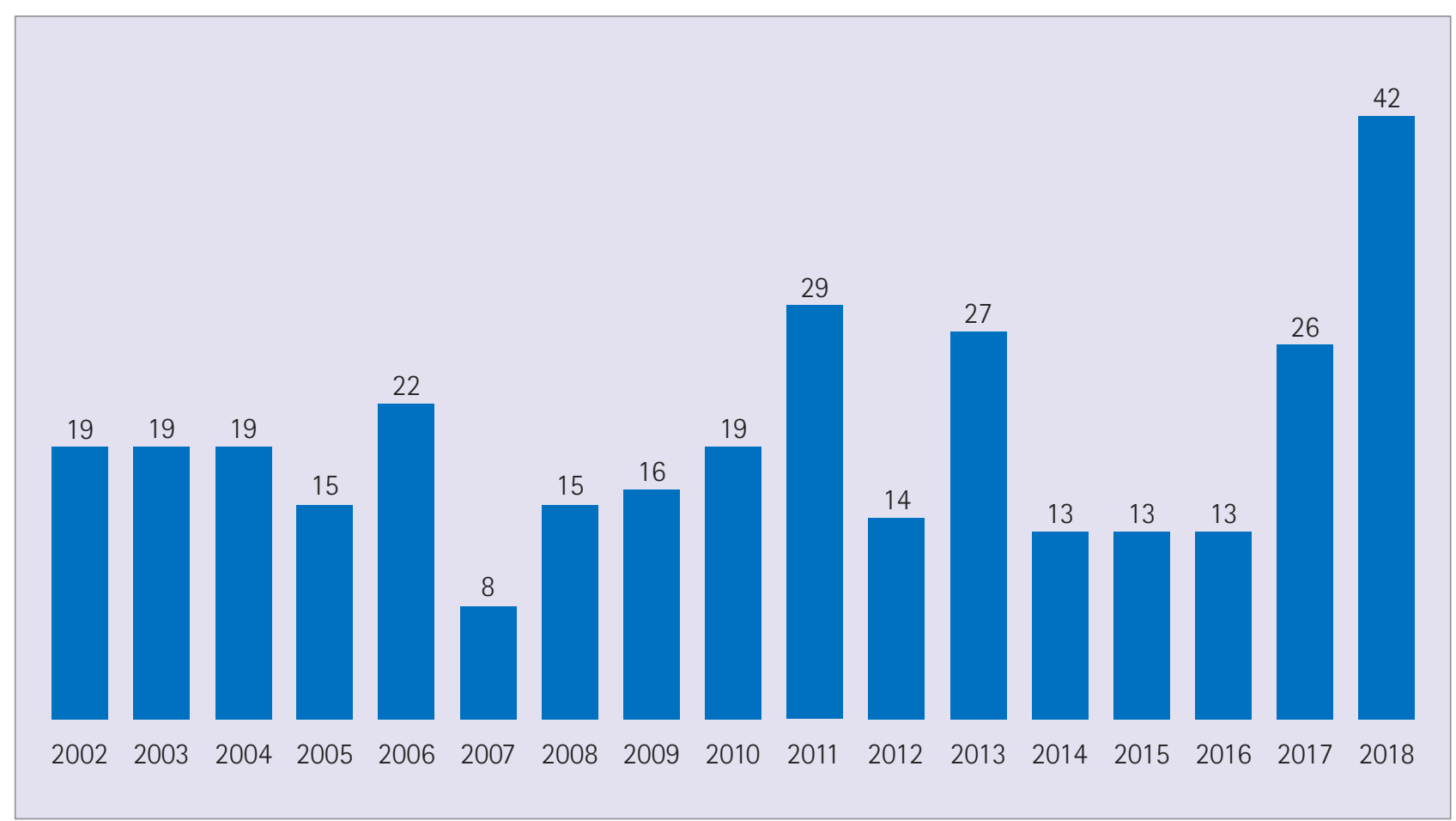

Figura 2 Número de RAM/año 
Tabla 3 Tipos de RAM

\begin{tabular}{|l|c|}
\hline Tipos de RAM & $\mathbf{n}(\%)$ \\
\hline $\begin{array}{l}\text { Neurológicas/ } \\
\text { Psicológicas }\end{array}$ & $140(31,7 \%)$ \\
\hline Digestivas & $99(22,4 \%)$ \\
\hline $\begin{array}{l}\text { Alérgicas/ } \\
\text { Dermatológicas }\end{array}$ & $63(14,3 \%)$ \\
\hline Osteomusculares & $41(9,3 \%)$ \\
\hline Cardiovasculares & $29(6,6 \%)$ \\
\hline Respiratorias & $21(4,8 \%)$ \\
\hline Genitourinarias & $18(4,0 \%)$ \\
\hline Oftalmológicas & $17(3,8 \%)$ \\
\hline Otras & $14(3,2 \%)$ \\
\hline
\end{tabular}

\section{Discusión}

Más de la mitad de las sospechas comunicadas se han registrado en pacientes mayores de 65 años. Es sobradamente conocida la elevada prevalencia de RAM en personas de mayor edad (11), siendo la polifarmacia y la complejidad de los tratamientos factores que contribuyen a la aparición de RAM (12). El riesgo aumenta con la edad, como consecuencia de los cambios fisiológicos del envejecimiento, los cambios en el comportamiento farmacocinético y farmacodinámico de los medicamentos $\mathrm{y}$ la influencia de las enfermedades, los problemas funcionales y los aspectos sociales (13).

El equipo farmacéutico debe continuar con el control más estrecho de los medicamentos en este grupo de población, especialmente en los de nueva comercialización, ya que suponen la mayoría de la población afectada por las RAM.

Se han detectado 12 sospechas en medicamentos publicitarios (3,6\%) y 7 en población pediátrica $(2,1 \%)$. Con el incremento actual de la disponibilidad medicamentos publicitarios podría ser necesario el diseño de programas específicos de farmacovigilancia, en los que la participación de la farmacia comunitaria puede resultar de gran importancia, al ser un punto estratégico desde el que se pueden identificar RAM de muchos medicamentos, incluidos los que no requieren prescripción médica y en el que la automedicación juega un papel importante en la aparición de RAM $(14,15)$.

Según las últimas estadísticas publicadas por el Centro Andaluz de
Farmacovigilancia, las notificaciones realizadas por farmacéuticos comunitarios durante el año 2018 han supuesto el 11,3\% del total de notificaciones efectuadas por los profesionales sanitarios (16). Este dato manifiesta que el grado de participación en el sistema de notificación de la farmacia comunitaria en Andalucía no es elevado; sobre todo si se compara con los porcentajes alcanzados por los farmacéuticos hospitalarios $(20,7 \%)$ y los médicos (60\%) (16). Puede ser necesario mejorar el acceso de la farmacia comunitaria a las actividades de farmacovigilancia, pues la mayoría de medicamentos se dispensan en las farmacias comunitarias y el farmacéutico comunitario, por su formación y proximidad, se encuentra en una posición favorable para implicarse más en la red de farmacovigilancia $(3,17)$.

Si estos datos se mantienen en toda la farmacia comunitaria española, podría ser conveniente que nuestros representantes profesionales diseñaran programas o buscaran iniciativas con el objetivo de incrementar la utilización del sistema de notificación espontánea de sospecha de RAM, ya que una de las funciones del farmacéutico es la de garantizar la seguridad de los medicamentos utilizados por la comunidad (12).

En la tabla 4 se muestra la representatividad de la farmacia de nuestro estudio respecto al número total de notificaciones de los profesionales sanitarios y el número de notificaciones de las farmacias comunitarias en Andalucía. La representatividad más alta de nuestra farmacia respecto a las notificaciones de las farmacias comunitarias andaluzas se ha obtenido en los años 2018 (43,8\%), 2017 (36,1\%) y 2011 $(31,4 \%)$.

Para nuestro sistema es importante que el registro de sospechas de RAM sea incorporado como un proceso sistemático en la dispensación de medicamentos. El proceso de detección de sospechas de RAM es entendido como una actividad de atención farmacéutica fundamental para mejorar los resultados del uso de los medicamentos. Para desarrollar estrategias para mejorar la calidad y la continuidad en las notificaciones espontáneas de nuestra farmacia, se ha intentado vencer algunas posibles "actitudes negativas" que llevan a los farmacéuticos del equipo a no notificar sospechas de RAM. Según un estudio realizado entre farmacéuticos comunitarios de Pontevedra estas actitudes fueron complacencia, ignorancia $\mathrm{y}$ subestimación (18). La complacencia se refiere a la convicción de que una vez se ha registrado un nuevo fármaco, este es totalmente seguro $\mathrm{y}$ eficaz. Al dispensar un medicamento, el farmacéutico comunitario puede enfrentarse en la práctica diaria

Tabla 4 Representatividad de la farmacia del estudio respecto a las notificaciones recibidas en el Centro Andaluz de Farmacovigilancia

\begin{tabular}{|c|c|c|c|}
\hline Año & $\begin{array}{c}\text { No notificaciones } \\
\text { Farmacia del } \\
\text { estudio }\end{array}$ & $\begin{array}{c}\text { Representatividad de la } \\
\text { farmacia del estudio sobre el } \\
\text { total de notificaciones }\end{array}$ & $\begin{array}{c}\text { Representatividad de } \\
\text { la farmacia del estudio } \\
\text { sobre las notificaciones de } \\
\text { farmacias andaluzas }\end{array}$ \\
\hline 2001 & 23 & $2,60 \%$ & Sin datos \\
\hline 2005 & 15 & $1,90 \%$ & $13,90 \%$ \\
\hline 2006 & 22 & $1,95 \%$ & $10,70 \%$ \\
\hline 2008 & 15 & $1,01 \%$ & $17,00 \%$ \\
\hline 2009 & 16 & $1,10 \%$ & $22,60 \%$ \\
\hline 2010 & 19 & $0,95 \%$ & $19,40 \%$ \\
\hline 2011 & 29 & $2,63 \%$ & $31,40 \%$ \\
\hline 2013 & 27 & $0,91 \%$ & $10,10 \%$ \\
\hline 2015 & 13 & $0,49 \%$ & $8,00 \%$ \\
\hline 2016 & 13 & $0,49 \%$ & $11,40 \%$ \\
\hline 2017 & 26 & $0,95 \%$ & $36,10 \%$ \\
\hline 2018 & 42 & $0,77 \%$ & $43,80 \%$ \\
\hline & & & $\%$
\end{tabular}

Fuente: https://www.cafv.es/centro-andaluz-de-farmacovigilancia/actividades/resumen-de-actividadespor-anos/) 
a situaciones nuevas y diferentes a la de los ensayos clínicos previos y puede sospechar RAM desconocidas o que, aun siendo conocidas, representen un aumento de la incidencia y/o de la gravedad (19).

Para potenciar actitudes positivas hacia la detección de sospechas de RAM se han llevado a cabo las siguientes intervenciones:

- Formación y actualización de la seguridad de los medicamentos mediante el estudio y registro de las notas y alertas de farmacovigilancia. El equipo farmacéutico actualiza periódicamente sus conocimientos sobre las notificaciones de seguridad recibidas en la farmacia. Además, se utiliza la herramienta informática $B O T+$, que permite incorporar mensajes de alerta específicos al consultar un fármaco (20).

- Incorporación de los registros de sospechas de RAM (tarjeta amarilla) en los objetivos de las actividades asistenciales de la farmacia. Se realiza un seguimiento del indicador del proceso de farmacovigilancia, número de tarjetas amarillas, mediante un sistema de gestión de la calidad según la norma ISO 9001.

- Elaboración periódica de una revisión bibliográfica sobre nuevos medicamentos por parte del equipo farmacéutico.

- La notificación on line en la página web del Centro Andaluz de Farmacovigilancia.

La media anual del número de tarjetas amarillas notificadas a lo largo del periodo del estudio $(19,4-\mathrm{DE}$ : $8,07)$ indica que el equipo farmacéutico ha asumido la notificación de sospechas de RAM dentro de las actividades asistenciales que tradicionalmente vienen llevando a cabo. Se podría considerar que en el desarrollo de este servicio vale la pena invertir tiempo y esfuerzo para contribuir a la utilización racional de los medicamentos.

Para asegurar la continuidad del servicio de farmacovigilancia, en nuestras dispensaciones se busca que nuestros pacientes nos informen de posibles efectos adversos, pero debemos evitar el efecto "nocebo"; que los pacientes le atribuyan sintomas adversos al medicamento por las expectativas negativas que ya tienen y abandonen el tratamiento por ese temor injustificado (21). De la misma forma que la expectativa de mejoría con respecto a un tratamiento puede generar un beneficio significativo, la expectativa de efectos adversos puede llevar a experimentar sintomas desagradables. Algunos estudios han demostrado que con una simple instrucción verbal se puede generar efecto "nocebo", y el farmacéutico debe ser consciente de que, aun de forma no intencionada, puede inducirlo, por lo que debe estar familiarizado con las estrategias para prevenirlo o minimizarlo. La información acerca de los potenciales efectos adversos debería ir acompañada de una explicación detallada sobre los efectos terapéuticos esperados (22).

Como es bien conocido, todo medicamento tiene sus efectos adversos, a la vez que el uso de medicamentos, incluido el uso correcto de los mismos, puede conllevar una probabilidad de aparición de problemas de seguridad (23). Es por ello que sería interesante implantar estrategias coordinadas entre atención primaria y especializada que permitan reducir riesgos de aparición de RAM, en las que se podrían incluir intervenciones como la revisión de la medicación por parte de un farmacéutico comunitario y seguimiento farmacoterapéutico de pacientes con mayor riesgo de sufrir reacciones adversas (12). La revisión de la medicación ha sido definida como la evaluación estructurada de los medicamentos de un paciente con el objetivo de optimizar el uso de medicamentos y mejorar los resultados de salud. Esto implica detectar Problemas Relacionados con Medicamentos (PRM) y recomendar intervenciones (24).

Aunque muchas de las RAM detectadas en nuestro estudio no sean graves, el farmacéutico comunitario debe estar atento a la detección de sospechas de RAM y evitarlas si es posible con la ayuda de protocolos de seguimiento farmacoterapéutico específicos (25). La actividad de farmacovigilancia debe ser entendida como un servicio orientado a evaluar y mejorar los resultados de la farmacoterapia, convirtiéndose en la puerta de entrada al servicio de seguimiento farmacoterapéutico (26).

Coincidiendo con otros estudios $(23,27,28)$, la terapia cardiovascular, los antibacterianos, los antiinflamatorios y los medicamentos para el sistema nervioso figuran entre los principales grupos farmacológicos implicados en la detección de sospechas de RAM.

Este estudio presenta como limitaciones que no analiza la relación de causalidad, pues está basado en la notificación espontánea de sospechas de RAM y que está desarrollado en una única farmacia comunitaria.

\section{Conclusiones}

Más de la mitad de las sospechas comunicadas se han registrado en pacientes mayores de 65 años. Aunque la mayoría de las RAM detectadas no sean graves, el equipo farmacéutico debe seguir atento a la detección de sospechas de RAM en este grupo de pacientes, especialmente en los medicamentos de nueva comercialización.

El análisis de las sospechas de RAM detectadas y comunicadas permite disponer de un conocimiento más completo de la seguridad del medicamento de los pacientes en nuestra actividad.

El servicio de farmacovigilancia se ha integrado en la sistemática diaria del equipo farmacéutico proporcionando un valor añadido en la farmacoterapia de los pacientes.

\section{Referencias bibliográficas}

1. Jimeno FJ, Salgueiro E, González V, Ganso G. Relevancia clínica de las notificaciones de reacciones adversas de atención primaria recibidas en el centro de farmacovigilancia de Asturias. Semergen. 2010; 36(2): 63-68. doi:10.1016/j.semerg.2009.12.001

2. Montpart E, Martín MP. El Sistema Español de Farmacovigilancia. Offarm 2003; 22 (2): 120-128.

3. Lorenzo B, Marcos L, Acuña A, Mera $\mathrm{R}$, Vérez N, Andrés Iglesias JC et al. Farmacovigilancia en farmacia comunitaria de medicamentos recientemente comercializados. Pharm Care Esp. 2015; 17(3):360-375. Disponible en: https://www.google.com/url? $\mathrm{sa}=\mathrm{tCtrct}=\mathrm{jCt} \mathrm{q}=\mathrm{Ctesrc}=\mathrm{sCt}$ ource $=$ we b\&tcd=ctved=2ahUKEwi4_IuP897rAhUWAGMBHa myAJcQFjAAegQIBRABCturl=https\%3A\%2F\%2Fwww. pharmcareesp.com\%2Findex.php\%2FPharmaCARE\%2Farticle\%2Fdownlo ad\%2F 23 5\%2F21 5 ctusg =AOvVaw1MtOrxTqMp0xSF8fBGzctD

4. Estudio APEAS. Estudio sobre la seguridad de los pacientes en atención primaria de salud. Madrid: Ministerio de Sanidad y Consumo; 2008. Disponible en: https://www.mscbs.gob. 
es/organizacion/sns/planCalidadSNS/ docs/estudio_apeas.pdf

5. Montané E, Santesmases J. Reacciones adversas a medicamentos. Med Clin (Barc). 2020; 154(5):178-184. doi:10.1016/j.medcli.2019.08.007

6. Montané E, Arellano AL, Sanz Y, Roca J, Farré M. Drug-related deaths in hospital in patients: A retrospective cohort study. Br J Clin Pharmacol. 2018; 84:542-52. doi:10.1111/bcp.13471

7. Real Decreto 577/2013, de 26 de julio, por el que se regula la farmacovigilancia de medicamentos de uso humano. BOE 2013, n. ${ }^{\circ} 179$ (27/07/2013), págs. 55.066-55.092. Disponible en: https://www.boe.es/buscar/doc.php?i$\mathrm{d}=$ BOE-A-2013-8191

8. Fletcher AP. Spontaneous adverse drug reaction reporting vs event monitoring: a comparison. J R Soc Med. 1991; 84: 341344. doi:10.1177/ 014107689108400612

9. Ley $44 / 2003$ de 21 de noviembre, de ordenación de las profesiones sanitarias. BOE, n. ${ }^{\circ} 280$ (22/11/2003), págs. 41.442-41.458. Disponible en: https:// www.boe.es/buscar/act.php?id=BOE-A-2003-21340

10. Ley $29 / 2006$ de 26 de julio, de garantías y uso racional de los medicamentos y productos sanitarios. BOE, n. ${ }^{\circ} 178$ (27/7/2006), págs. 28.122-28.165. Disponible en: https://www.boe.es/buscar/act.php?id=BOE-A-2006-13554

11. Molina MJ, Napal V. Análisis de las notificaciones recibidas en la unidad de farmacovigilancia de la Sociedad Española de Farmacia Hospitalaria 19901998. Farm Hosp. 1999; 23(3): 191-197. Disponible en: https://www.elsevier. es/en-revista-farmacia-hospitalaria-121-articulo-analisis-de-las-notificaciones-recibidas- 13005188

12. Sánchez M, Delgado L, Delgado E, Prieto S, Bermejo T. Detección y análisis de reacciones adversas a medicamentos en el servicio de urgencias de un hospital general. Farm Hosp. 2006; 30(2): 78-84. doi:10.1016/S11306343(06)73951-4

13. Delgado E, Muñoz M, Montero B, Sánchez C, Gallagher PF, Cruz-Jentoft AJ. Prescripción inapropiada de medicamentos en los pacientes mayores: los criterios STOPP/START. Rev Esp Geriatr Gerontol. 2009; 44(5): 273279. doi:10.1016/j.regg.2009.03.017
14. Medina MA, Puche E, de Dios Luna J. Factores asociados con la presentación de reacciones adversas a medicamentos en pacientes que acuden al servicio de urgencia de un hospital general: estudio de casos y controles. Aten Primaria 2000; 26(1): 42-4. doi:10.1016/S0212-6567(00)78605-7

15. Layton D, Sinclair HK, Bond CM, Hannaford PC, Shakir SAW. Pharmacovigilance of over-the-counter products based in community pharmacy: methodological issues from pilot work conducted in Hampshire and Grampian, UK. Pharmacoepidemilogy and Drug Safety. 2002; 11: 503-513. doi:10.1002/pds.734

16. Centro Andaluz de Farmacovigilancia [Internet]. Notificaciones por profesionales sanitarios 2018. [Acceso 30/04/2020]. Disponible en: https://www.cafv.es/wp-content/ uploads/2020/02/MEMORIA-2018_PARA-WEB.pdf

17. Tena T, Rivera A, Beas AI, Alonso C, Bravo E, Rodríguez R. Farmacovigilancia: ¿y si notificamos? Farmacéuticos comunitarios. 2014; 6(2): 4852. doi:10.5672/FC.2173-9218.(2014/ Vol6).002.07

18. Acuña A, González D, Castillo A, Fornos JA, Andrés JC, Andrés NF. Metodología para evaluar las actitudes y aptitudes sobre farmacovigilancia en los farmacéuticos comunitarios. Aplicación en la provincia de Pontevedra. Pharm Care Esp. 2012; 14 (3): 110-121. Disponible en: https://www.google. com/url? sa $=$ tEtrct $=$ jEtq $=$ Etesrc $=$ sEt source $=$ webctcd $=$ etved $=2$ ahUKEwiZ n9inld_rAhUFAGMBHTjjBYYQFjAAegQIBhABCturl=https $\% 3 \mathrm{~A} \% 2 \mathrm{~F} \%-$ 2Fwww.pharmcareesp.com\%2Findex. php\%2FPharmaCARE\%2Farticle\%2Fdownload\%2F75\%2F69ctusg=A0vVaw0FGkUK3pUagNcXw-TjnrRD

19. Borja J, Souto M. Importancia de la farmacovigilancia en la práctica de médico de familia. Semergen. 2006; 32(7): 334-8. doi:10.1016/S1138-3593 (06)73285-3

20. Hidalgo T, Cuéllar S, Megia C, Varas R, Martín L, Torres A et al. Pilotaje de un procedimiento de farmacovigilancia. Farmacéuticos Comunitarios. 2009; 1(2): 68-70. Disponible en: https:// www.farmaceuticoscomunitarios. org/system/files/journals/111/articles/01-3-68-70.pdf

21. Cuéllar S. Placebo y nocebo. Panorama Actual Med. 2019; 43 (421): 187-205. Disponible en: http://pam.portalfarma. com/placebo-y-nocebo/

22. ¿Qué sabemos sobre el efecto placebo? Infac. 2015; 23 (8). Disponible en: http://www.euskadi.eus/contenidos/informacion/cevime_infac_2015/ es_def/adjuntos/INFAC_Vol_23_N_8_ placebo.pdf

23. Alos M, Bonet M. Análisis retrospectivo de los acontecimientos adversos por medicamentos en pacientes ancianos en un centro de salud de atención primaria. Aten Primaria. 2008; 40 (2): 75-80. doi:10.1157/13116153

24. Pharmaceutical Care Network Europe [internet]. The netherlands: PCNE; 2010-2016 [consultado mayo 2020]. Disponible en: http://www. pcne.org/working-groups/1/medicationreview

25. Barris D. Sospechas de reacciones adversas a medicamentos notificadas en una farmacia comunitaria. Pharm Care Esp. 2002; 4: 250-251.

26. Foro de Atención Farmacéutica-Farmacia Comunitaria (Foro AF-FC). Guía Práctica para los Servicios Profesionales Farmacéuticos Asistenciales en la Farmacia Comunitaria. Madrid: Consejo General de Colegios Oficiales de Farmacéuticos; 2019. https://www.portalfarma.com/inicio/serviciosprofesionales/forofarmaciacomunitaria/ Documents/2019-guia-practica-spfa.pdf

27. Yu YM, Shin WG, Lee J-Y, Choi SA, Jo YH, Youn SJ, et al. (2015) Patterns of Adverse Drug Reactions in Different Age Groups: Analysis of Spontaneous Reports by Community Pharmacists. PLoS ONE 10(7): e0132916. doi:10.1371/journal.pone. 0132916

28. Pérez-Ricart A, Gea-Rodríguez E, Roca-Montañana A, Gil-Máñez E, Pérez-Feliu A. Integración de la farmacovigilancia en la rutina del servicio de farmacia: nueve años de experiencia. Farm Hosp. 2019; 43(4):128-133. Disponible en: https://revistafarmaciahospitalaria.sefh.es/gdcr/index. php/fh/article/view/11169 